\title{
A MULTI-BANDED SYSTEM ARCHITECTURE FOR ULTRA-WIDEBAND COMMUNICATIONS
}

\author{
Jeffrey R. Foerster, V. Somayazulu, and Sumit Roy \\ Intel Research and Development \\ 2111 N.E. $25^{\text {th }}$ Ave., Hillsboro, OR 97124-5961 \\ jeffrey.r.foerster@intel.com
}

\begin{abstract}
The FCC ruling in 2002 allowing for the unlicensed deployment of ultra-wideband (UWB) devices for communications purposes in the $3.1-10.6 \mathrm{GHz}$ band has sparked great interest in the industry. In particular, the IEEE 802.15.3a task group is currently developing a standard for high-rate, short-range wireless communication systems that is expected to use UWB technology. One of the main challenges for UWB systern design is minimizing the possible interference to other narrowband systems, while, at the same time, dealing with the large interference that may be coming from these narrowband systems into the UWB receiver. Traditional UWB systems have used very short time impulses that occupy several giga-hertz of bandwidth. This approach makes it difficult to efficiently avoid other system that may be sharing the same band. This paper will describe a channel model that has been adopted by the industry to evaluate the merits of different UWB physical layer approaches and introduce an alternate approach to a high-rate UWB system that is based upon the concatenation of multiple ënarroweri band UWB waveforms.
\end{abstract}

\section{INTRODUCTION}

UWB system design has many challenges. These systems need to operate in indoor channels that tend to have severe multipath propagation. The very wide spectrum that UWB systems have been allocated results in many possible interferers that may be present at the receiver. In addition, since UWB systems will overlay other narrowband systems, it much demonstrate that ability to peacefully coexist, which may result in reducing power in certain parts of the band at the transmitter (most notably, coexistence with 802.11 a systems in the $5 \mathrm{GHz}$ band). Finally, the target applications that many people in the industry envision for UWB systems are for hand-held, mobile devices which require lower power consumption and low cost implementations. Traditional impulse radio techniques using multi-GHz bandwidth waveforms have advantages in propagating through multipath channels, but suffer from limited ability to avoid other interferers without using RF notch filters which may be difficult to integrate into a cheap CMOS process. This paper describes an alternate multi-band approach to UWB system design that uses a concatenation of multiple narrower band waveforms rather than a single ultra- wideband waveform. As will be discussed, this has the benefit of being able to peacefully coexist with both known and unknown narrow band systems that may be colocated with the UWB device, as well as adapt to possibly different worldwide spectrum regulations. Since itís important to understand the channel characteristics for any wireless system design, the next section describes a channel model that was derived from a series of measurements. A slightly modified model is also described that has been adopted by the IEEE 802.15.3a task group to evaluate possible UWB physical layer proposals for short-range, wireless personal area networks (WPANs). Then, a detailed description of a multi-band system approach is provided in the following section, which addresses coding, modulation, multipath access techniques, multipath mitigation techniques, coexistence, and implementation issues. Finally, concluding remarks are provided in Section 4.

\section{UWB CHANNEL MODELING}

In order to do proper system design, and understand and quantify the impact of multipath propagation, itis important to have a reliable channel model that captures the important characteristics of the channel. Towards this end, we have evaluated a number of popular indoor channel models to determine which model best fits the important characteristics that were measured and documented in [2]. The analysis and results of this channel modeling work have been submitted to the IEEE 802.15.3a channel modeling sub-committee [3], but are summarized here for completeness. Three indoor channel models were considered, the tap-delay line Rayleigh fading model [4], the Saleh-Valenzuela (S-V) model [5], and the $\Delta-\mathrm{K}$ model described in [6]. Each channel model was parameterized in order to best fit the important channel characteristics, which included the mean excess delay, mean RMS delay, and mean number of significant paths defined as paths within $10 \mathrm{~dB}$ of the peak path power. Our results found that the $\mathrm{S}-\mathrm{V}$ model was able to best fit the channel measurements and observed characteristics of the channel. In particular, the channel measurements showed a clustering of the multipath arrivals, which is also found in [7] and captured by the S-V model. In addition, the amplitude statistics of the measurements was found to best fit the log-normal distribution rather than the Rayleigh, which was part of the original S-V model. We have since compared the 
amplitude distribution to the Nakagami distribution and found that both the log-normal and Nakagami distributions fit the data equally well. So, the S-V model was modified slightly in order to take the log-normal fading distribution into account. The final proposed model is described next (see [3] for more details).

The proposed multipath model consists of the following, discrete time impulse response:

$$
h(t)=\sum_{l=0}^{L} \sum_{k=0}^{K} \alpha_{k, l} \delta\left(t-T_{l}-\tau_{k, l}\right)
$$

where $\alpha_{k, l}$ are the real multipath gain coefficient, $T_{l}$, is the delay of the $l^{t h}$ cluster, and $\tau_{k, l}$ is the delay of the $k^{\text {th }}$ multipath component relative to the $l^{\text {th }}$ cluster arrival time $\left(T_{l}\right)$.

Finally, the proposed model uses the following definitions (similar to [5]):

$T_{l}=$ the arrival time of the first path of the $l$-th cluster;

$\tau_{k, l}=$ the delay of the $k$-the path within the $l$-th cluster relative to the first path arrival time, $T_{l}$;

$\Lambda=$ cluster arrival rate;

$\lambda=$ ray arrival rate, i.e., the arrival rate of paths within each cluster.

By definition, we have $\tau_{0 l}=T_{l}$. The distribution of cluster arrival time and the ray arrival time are given by $p\left(T_{l} \mid T_{l-1}\right)=\Lambda \exp \left[-\Lambda\left(T_{l}-T_{l-1}\right)\right], \quad l>0$ $p\left(\tau_{k, l} \mid \tau_{(k-1), l}\right)=\lambda \exp \left[-\lambda\left(\tau_{k, l}-\tau_{(k-1) l}\right)\right], \quad k>0$

The channel coefficients are then defined as follows: $\alpha_{k, l}=p_{k, l} \beta_{k, l}$ $20 \log 10\left(\beta_{k, l}\right) \propto \operatorname{Normal}\left(\mu_{k, l}, \sigma^{2}\right)$, \{or $\left|\beta_{k, l}\right|=10^{n / 20}$ where $\left.n \propto \operatorname{Normal}\left(\mu_{k, l}, \sigma^{2}\right) \quad\right\}$ $E\left[\beta_{k, l}^{2}\right]=\Omega_{0} e^{-T_{l} / \Gamma} e^{-\tau_{k, l} / \gamma}$

where $T_{l}$ is the excess delay of cluster $l$ and $\Omega_{0}$ is the mean power of the first path of the first cluster, and $p_{k, l}$ is equiprobable $+/-1$. The $\mu_{i}$ term is given by $\mu_{l}=\frac{10 \ln \left(\Omega_{0}\right)-10 T_{l} / \Gamma-10 \tau_{k, l} / \gamma}{\ln (10)}-\frac{\sigma^{2} \ln (10)}{20}$.

The proposed model was used to fit Intelís measurements, and the following table provides the results of this fit for a couple of different channel scenarios (LOS refers to lineof-sight, and NLOS refers to non-LOS). Note that, when using the model, the total average received power of the multipath realizations is typically normalized to one in order to provide a fair comparison with other wideband and narrowband systems. This can be done either by normalizing each realization or by normalizing the total power, averaged over all realizations. The channel characteristics and corresponding parameter matching results in the table below correspond to a time resolution of $167 \mathrm{psec}$.

\begin{tabular}{|l|l|l|l|}
\hline $\begin{array}{l}\text { Channel } \\
\text { Characteristics }\end{array}$ & LOS & NLOS & $\begin{array}{l}\text { LOS+ } \\
\text { NLOS }\end{array}$ \\
\hline $\begin{array}{l}\text { Mean excess delay } \\
(\mathrm{nsec})\left(\tau_{m}\right)\end{array}$ & 4 & 17 & 14 \\
\hline $\begin{array}{l}\mathrm{RMS} \text { delay (nsec) } \\
\left(\tau_{r m s}\right)\end{array}$ & 9 & 15 & 13 \\
\hline $\mathrm{NP}_{10 \mathrm{~dB}}$ & 7 & 35 & 33 \\
\hline Model Parameters & & & \\
\hline$\Lambda$ (1/nsec) & $1 / 60$ & $1 / 11$ & $1 / 13$ \\
\hline$\lambda(1 / \mathrm{nsec})$ & $1 / 0.5$ & $1 / 0.35$ & $1 / 0.23$ \\
\hline$\Gamma$ & 16 & 16 & 13 \\
\hline$\gamma$ & 1.6 & 8.5 & 6 \\
\hline$\sigma(\mathrm{dB})$ & 4.8 & 4.8 & 4.8 \\
\hline
\end{tabular}

Table 1: Multipath channel characteristics and model parameters.

Some important characteristics of the multipath channel are important to discuss. First, the multipath spans several nano-seconds in time which results in inter-symbol interference (ISI) if UWB pulses are closely spaced in time. However, this interference can be mitigated in a number of ways through proper waveform design and signal processing and equalization algorithms. Second, the very wide bandwidth of a transmitted pulse results in the ability to individually resolve several multipath components. This is good and bad. It is good in the sense that the multipath arrivals will undergo less amplitude fluctuations (fading) since there will be fewer reflections that cause destructive/constructive interference within the resolution time of the received impulse. On the other hand, the average total received energy is distributed between a number of multipath arrivals. In order to take advantage of that energy, unique systems and receivers need to be designed with multipath energy capture in mind. For a traditional impulse based UWB waveform, this may consist of a rake receiver with multiple arms, one for each resolvable multipath component. However, as the bandwidth of the UWB waveform increases, the complexity of the RAKE receiver could become limiting in order to capture the same energy. As a result, careful bandwidth selection of the UWB waveform can help balance the receiver complexity. for capturing multipath 
energy while still benefiting from the reduced fading of the short duration of the pulses.

Finally, itis worth noting the channel modeling work done in the IEEE 802.15.SG3a standards committee, where a final report can be viewed in [9] that is based upon some slight modifications of the above model. The key area that was changed from the above model includes the following:

- Separation between cluster and ray fading: In the above model, the fading on a multipath arrival is based upon a single log-normal distribution with the power decay given by the double exponential profile defined above. In the revised model in [9], the fading on a multipath arrival is given by the product of a lognormal fading term associated with each cluster and an independent log-normal fading term associated with the ray with the cluster. This change was made to better match visual inspections of actual channel impulse responses.

Although the work of the IEEE 802.15.3a channel modeling sub-committee was intended to help evaluate physical layer proposals to the IEEE 802.15.SG3a committee, much more work remains on the characterization of UWB pulse propagation as a function of pulse bandwidth and operating environment. Many other contributions have also been submitted to the IEEE 802.15.3a channel modeling sub-committee work which describe measurements and models for a number of different environment. In particular, correlation of different model parameters for different pulse bandwidths in different environments still requires more measurements. In addition, an understanding of the timevariation expected for short-range wireless channel, either due to the movement of the UWB devices or of people or objects within the area of the UWB device, would be very useful for optimize a UWB physical layer.

\section{A MULTI-BANDED SYSTEM ARCHITECTURE}

In order to address several of the challenges discussed in the previous sections, we have moved away from the traditional impulse based radio design in favor of a multibanded radio design. In particular, the key idea is to divide the spectrum in a number of ënarroweri bands in order to provide some spectrum flexibility, and concatenate these bands in a manner that provides good performance in multipath and good multiple access protection. There are many possible ways to divide the spectrum and architect a system that can take advantage of this flexibility, as seen in many different proposals to the IEEE 802.15.3a task group, so this section only focuses on one approach pursued by Intel, and discusses a number of trade-offs that exist when designing this kind of system.
Figure 1 shows an example time and frequency domain representation of a multi-band waveform based upon the transmission of non-overlapping sub-band pulses, where each band has a bandwidth of about $500 \mathrm{MHz}$. In this example, the center frequency of each pulse is changed on a pulse-by-pulse basis, and the concatenation of these pulses occupy about $4 \mathrm{GHz}$ of spectrum. For explanation purposes, this example is referred to as a length 1 extended approach, where the center frequency is changed after dwelling on a particular frequency for 1 symbol. The following sub-sections revisit this method to investigate the merits of dwelling on a frequency for more than one symbol period, and will describe in more detail a particular system architecture initially pursued by Intel and proposed to the IEEE 802.15.3a task group. Note that we continue to look for improvements and continue to work with the IEEE 802.15.3a task group towards a common UWB physical layer, which, in the end, may look very different from the system described below. As a result, we will focus the discussion below more on qualitative system trade-offs rather than quantitative performance analysis. The system below has been simulated extensively in the presence of multipath and multiple access interference, and the results are provided in [8] for interested readers.

\section{a. Modulation, coding, and waveform shape}

Figure 2 shows the transmiter and receiver block diagrams for the proposed system. Since UWB system are power limited rather than spectrum limited, itis important to use very power efficient modulation schemes. As a result, we have chosen to use M-ary, bi-orthogonal keying (MBOK) with BPSK and QPSK symbols. An outer $(255,221)$ Reed-Solomon (RS) code is used to correct bursts of errors that result from the MBOK decoder. In AWGN, this coding and modulation scheme yields a required $\mathrm{Eb} / \mathrm{No}=$ $3.5 \mathrm{~dB}$ for a packet error rate of less than $8 \%$. In addition, the use of MBOK enables efficient symbol based equalization techniques, where reliable MBOK decisions could be used in a decision feedback equalizer to help mitigate ISI prior to RS decoding. Variable data rates are also supported through the use of variable MBOK mappings. For example, a mapping of 6 bits into a length 32 Walsh-Hadamard sequence results in a rate $6 / 32$ code, mapping 5 bits into a length 16 sequence results in a rate $5 / 16$ code, etc. Note that, alternate coding schemes could also be used, like low rate convolutional codes, turbo codes, or concatenated codes. The main trade-offs that must be considered, as with any communication system design, include decoder complexity, power efficiency/performance of code, and equalization complexity.

A sub-band waveform period of 3 nsec using a rectified cosine shape what chosen, which yields a sub-band bandwidth of about $700 \mathrm{MHz}$ and results in a total of 13 
bands between 3.1 and $10.6 \mathrm{GHz}$. The slightly wider bandwidth (compared to a $500 \mathrm{MHz}$ waveform) allows for a lower rate code to be used for a given data rate. This lower rate code provides greater robustness in the presence of multipath and multiple access interference and, at the same time, maintains good spectrum flexibility, as will be discussed below. For example, a 221/255 RS code with a $6 / 32 \mathrm{MBOK}$ code and $3 \mathrm{nsec}$ symbol period using both 1 and $Q$ channels yields a data rate of $108 \mathrm{Mbps}$. A 5/16 MBOK code yields a data rate of $180 \mathrm{Mbps}$, a $4 / 8 \mathrm{MBOK}$ code yields a data rate of $288 \mathrm{Mbps}$, and a $æ$ MBOK code yields a data rate of $433 \mathrm{Mbps}$. The proper selection of symbol bandwidth has a number of implications in terms of spectrum flexibility, and performance in multipath and multiple access interference, as will be discussed below.

Finally, Figure 3 shows how the MBOK chips are mapped in time and frequency. In this example, each frequency is ëoni for a period of 4 symbols (referred to as a length 4 extension), which has some advantages in mitigating multiple access and multipath interference. In addition, the MBOK chips are interleaved in a manner that allows for a possible DFE to be used to help mitigate the ICI that would be caused by multipath, as discussed below.

\section{b. Channelization and multiple access techniques}

The applications envisioned for UWB enabled devices are wide ranging, and include mobile (cellular), consumer electronic (CE), and personal computer (PC) market segments. As a result, there may be many devices in close proximity that will want to communicate with each other in either an ad hoc basis or through a ëpiconeti coordinator. The IEEE $802.15 .3 \mathrm{a}$ task group is currently planning on using the 802.15.3 MAC, which uses a TDMA protocol for sharing the media between locally connected and coordinated devices. In addition, methods must be available to allow for multiple, uncoordinated devices to also share the same location. This requirement could result in devices that are closely located to each other sharing the same spectrum and causing possible severe near-far problems. As a result, a number of different channelization methods may be useful to accommodate different sharing environments.

In this paper, we describe a multiple access scheme that uses a hybrid direct sequence (DS) and frequency hopping (FH) CDMA system with an FDMA back-off mode to handle situations with a severe near-far problem. In Figure 2 , the random PN mask, in conjunction with a low-rate code discussed in the previous section provides some separation between different users (this is the DS component). In Figure 3, a plot of the transmitted frequencies in time is shown. If different users transmitted a different order of the frequencies, this provides an additional level of separation (this is the $\mathrm{FH}$ component).
In particular, ( $\left.p^{\wedge} \mathrm{m}-1\right) \mathrm{FH}$ codes exist, using primitive polynomials over $G F\left(p^{\wedge} m\right)$ for some prime number $p$ and integer $\mathrm{m}$, which result in hopping patterns with a ëonecoincidencei property. This means that, for any two sequences and any delay between the two sequences, one and only one frequency will be colliding. For example, for a frame period based upon the serial concatenation of 7 sub-bands as shown in Figure 3, six different FH codes exist with this ëone-coincidenceí property. So, when little or no multipath exists between the interfering and desired user, only 1 of 7 bands will be comupted which the code must try to overcome. Of course, multipath between the interfering user and the desired receiver will cause multiple frequencies to collide and slowly degrade performance, which is why the combination of DS and FH are needed. The length 4 extension discussed previously helps reduce the number of frequency collisions by extending the overall frame duration. Finally, in situations where even greater isolation between piconets is needed, the piconets could coordinate the sharing of the bands, using mechanisms that already exist in the 802.15.3 MAC, essentially enabling a form of FDMA. In addition, dividing the spectrum into 13 bands total allows for different piconets to use different parts of the spectrum as needed.

\section{c. Multipath mitigation methods}

There are several trade-offs that exists when selecting the proper waveform shape and bandwidth. UWB impulse radio systems are well known for their ability to finely resolve multipath reflections and experience much less fading than traditional narrowband waveforms. So, is moving toward waveforms with ënarroweri bandwidths going to throw away the advantages of UWB systems? Not necessarily, since other forms of diversity can help mitigate the effect of the increased fading. Figure $4 \mathrm{a}$ shows the greater fading, as expected, experienced by narrower band waveforms based upon the channel models adopted by the IEEE 802.15.3a group. However, Figure $4 \mathrm{~b}$ shows that, on average, more energy can be captured by a single arm rake by averaging the total received power over multiple frequency bands compared to a single wideband impulse. Both systems can also take advantage of path diversity using more rake arms, but the wider bands system would need more rake arms to capture the same amount of energy as the narrower band implmentation.

There are four main tools that help mitigate and take advantage of multipath propagation:

- Interleaving the MBOK chips over the different frequencies, as shown in Figure 3, allows the receiver to take advantage of the frequency diversity experienced by independent fades on each frequency. 
- The fixed FH pattern and the length 4 extension method ensures that the groups of 4 symbols on each frequency are separated by $72 \mathrm{nsec}$. This allows the channel to ring down before the next set of chips on the same frequency are transmitted and reduces ISI. The waveform shape helps reduce adjacent channel interference when multipath causes adjacent bands to overlap.

- The ICI within the 4 chip ëoní period does require equalization to help mitigate. This is supported, in part, by the interleaving technique shown in Figure 3 which allows MBOK decisions to be fed back into a DFE to cancel the self interference caused by the channel. This could be accomplished in a single iteration, or multiple iterations in the receiver.

- Finally, the 4 symbol ëoni period for each frequency allows for a 4-tap rake for each symbol to help capture more channel energy. Since each sample will have both desired symbol energy as well as interfering symbol energy, an MMSE or MLSE based receiver would be useful to balance energy capture with increased self interference. Limiting the ëoni period to only 4 symbols keeps the complexity of these schemes reasonable. In addition, a second parallel receiver could help rake in energy that spans 8 symbol periods, or 24 nsec, which should be sufficient for many multipath channels.

\section{d. Coexistence and narrowband interference mitigation}

Dividing the spectrum into a number of narrower bands yields some flexibility in avoiding narrowband interference at both the transmitter and receiver. In some cases, the interference is known, like the existence of 802.11 a systems in the $5 \mathrm{GHz}$ band, and in other cases, interferers may be unknown as future spectrum allocations may change. The previously described multi-band approach allows for both static techniques of avoiding certain bands where known narrowband systems exist as well as dynamic techniques where bands can be avoided only when the presence of interference is detected. There are also other important design considerations to take into account when requiring operation near strong interferers. In particular, the analog front-end of the receiver must be designed to prevent being driven into saturation by these interferers. The linear dynamic range of the LNA and front-end mixers, for example, becomes an important design parameter to ensure proper operation in the presence of strong interference even though certain bands are being avoided at the transmitter. This is a common challenge for any UWB system and is not unique to a multi-band implementation.

\section{e. Implementation feasibility}

Clearly, cost and power consumption are also important characteristics for any UWB system, based upon the target applications and use in hand-held, mobile devices. The main challenge for making this multi-band system work is the ability to generate and rapidly switch between multiple center frequencies. This is an active area of circuits research, but initial approaches look promising which do not consume much more power than a single PLL (i.e., a bank of PLLs are not necessary to generate these different center frequencies since only one at a time is being used). The ability to turn off some or all of the frequencies when not being used becomes important in order to keep the power consumption low. Having the bands transmit and receive in non-overlapping intervals helps simplify some implementations and allows many components to be shared (i.e., an independent receiver for each band is not required). However, rake reception may benefit from multiple parallel branches in the receiver to improve performance in severe multipath channels.

\section{CONCLUSIONS}

This paper provides a high level overview of a new multibanded approach to UWB systems that seems to have some useful properties. In particular, given the large allocation of frequency over which UWB systems are allowed to operate, the ability to detect and avoid interference seems critical. By dividing the spectrum into a number of narrower sub-bands, both known and unknown interferers could be avoided at both the transmitter and receiver. In addition, the uncertainty over worldwide regulations may require devices to adapt spectrum use to different geographical regions. However, there exists several areas for improvement that merit further investigation, including efficient equalization algorithms that can both rake in desired energy and mitigate self interference caused by multipath, interference detection and avoidance algorithms, and low cost circuit implementations that can efficiently implement a multiband type radio.

\section{REFERENCES}

[1] J. Proakis, Digital Communications Fourth Edition, McGraw-Hill Book Company, New York, 2001.

[2] L. Rusch, et. al, i Characterization of UWB Propagation from 2 to $8 \mathrm{GHz}$ in a Residential Environmentî, submitted for publication.

[3] J. Foerster and Q. Li, i UWB Channel Modeling Contribution from Intel,î IEEE P802.15-02/279-SG3a.

[4] IEEE 802.11-97/96, Naftali Chayat, September 1997.

[5] A. Saleh and R. Valenzuela, ì A Statistical Model for Indoor Multipath Propagation,î IEEE JSAC, Vol. SAC-5, No. 2, Feb. 1987, pp. 128-13.7.

[6] H. Hashemi, ì Impulse Response Modeling of Indoor Radio Propagation Channels,î IEEE JSAC, Vol. 11, 
No. 7, Sept. 1993, pp. 967-978.

[7 ] J.M. Cramer, R.A. Scholtz, and M.Z. Win, i On the analysis of UWB communication channel,î

Proc. of MILCOM 1999, Vol. 2, pp. 1191-1195, 1999.

[8] J. Foerster, et.al., i Intelis UWB PHY Proposal for IEEE 802.15.3a,î IEEE802.15-03/109(see http://ieee802.org/15/)

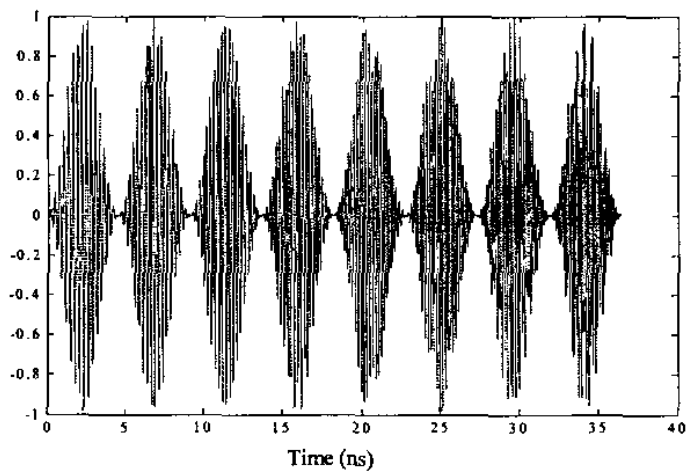

(a)

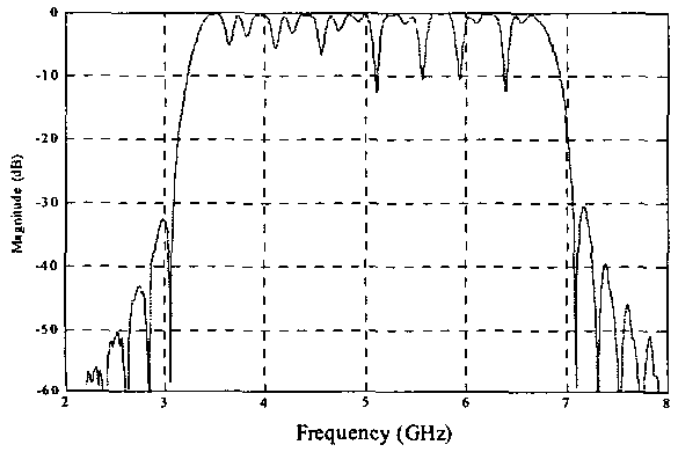

(b)

Figure 1. Example (a) time and (b) frequency domain representations of a multi-band waveform based upon a non-overlapping concatenation of multiple sub-bands.

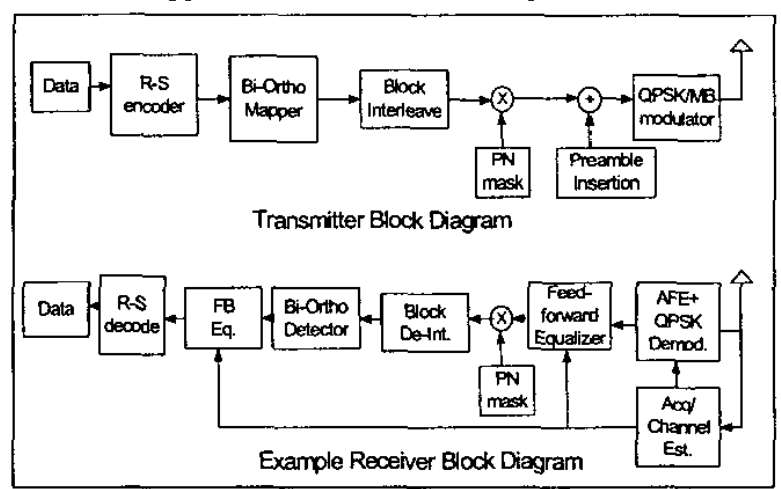

Figure 2. Example transmitter and receiver block diagram for a multi-banded radio.

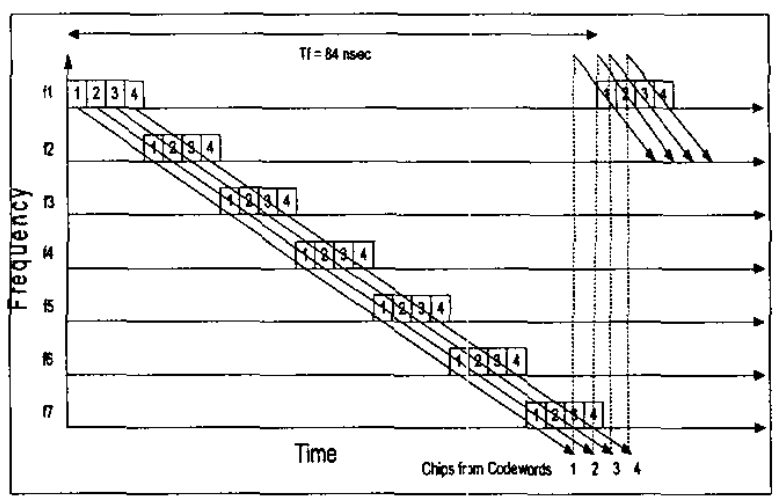

Figure 3. Time and frequency interleaving of data to support decision feedback equalization.

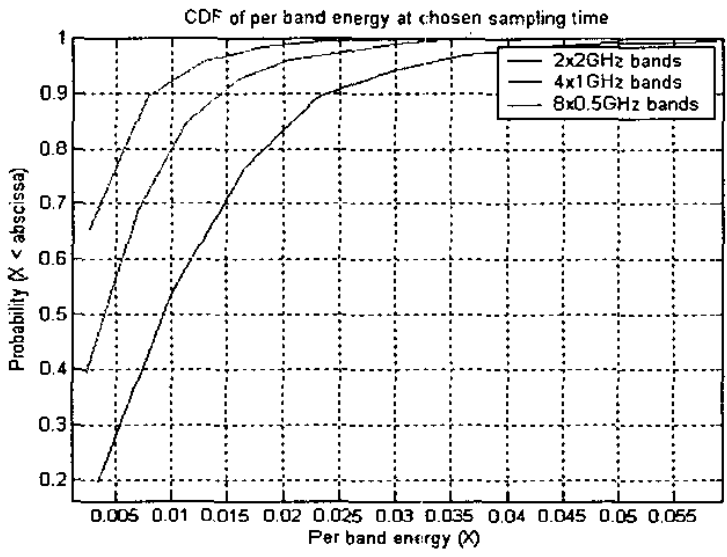

(a)

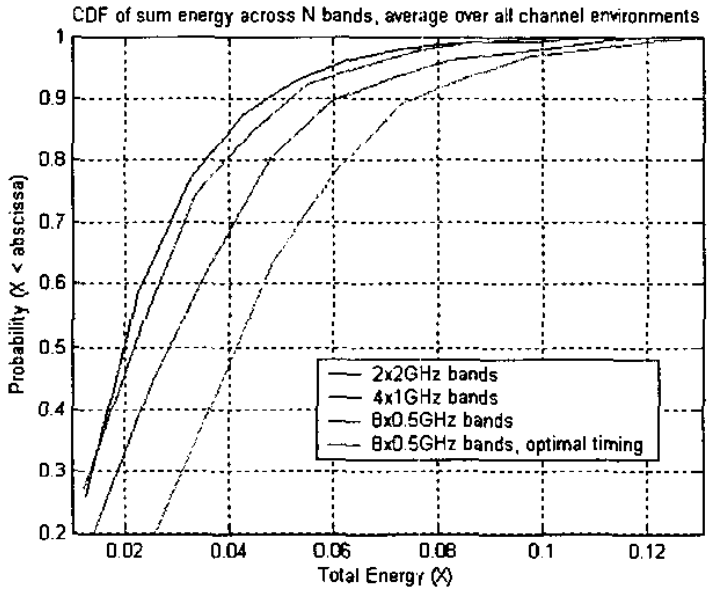

(b)

Figure 4. Amplitude variation (a) and total energy capture

(b) characteristics of the channels as a function of bandwidth for a single arm rake. 